\title{
Cardiomyopathies among Children Attending a Tertiary Hospital in South- western Nigeria
}

\author{
Animasahun $\mathrm{BA}^{* 1}$, Ogunkunle $\mathrm{OO}^{2}$, Madise-Wobo $\mathrm{AD}^{3}$, Gbelee $\mathrm{OH}^{1}$ and Oke $\mathrm{DA}^{4}$ \\ ${ }^{1}$ Department of Pediatrics and Child Health, Lagos State University College of Medicine, Ikeja, Lagos, Nigeria \\ ${ }^{2}$ Department of Paediatrics and Child Health, University College Hospital, Ibadan, Oyo State, Nigeria \\ ${ }^{3}$ Department of Paediatrics, Lagos State University Teaching Hospital, Ikeja, Lagos, Nigeria \\ ${ }^{4}$ Department of Medicine, Lagos State University Teaching Hospital, Ikeja, Lagos, Nigeria
}

*Corresponding author: Animasahun BA, Department of Paediatrics and Child Health, Lagos State University College of Medicine, Ikeja, Lagos, Nigeria, Tel: +2348037250264, E-mail: deoladebo@yahoo.com

Citation: Animasahun BA, Madise-Wobo AD, Ogunkunle OO, Gbelee OH, Oke DA (2015) Cardiomyopathies among Children Attending a Tertiary Hospital in South-western Nigeria. J Clin Exp Res Cardiol 2(3): 302. doi: 10.15744/2394-6504.2.302

Received Date: September 25, 2015 Accepted Date: November 26, 2015 Published Date: November 30, 2015

\begin{abstract}
Background: Acquired heart diseases such as myocarditis, cardiomyopathies and rheumatic heart diseases are important noncommunicable diseases responsible for morbidity and mortality in children. We aim to describe the pattern and distribution of cardiomyopathies in children less than 13 years in a tertiary hospital in Southwestern Nigeria and compare with patterns in the subregion.

Methods: Prospective and cross sectional, involving consecutive patients diagnosed with cardiomyopathy using echocardiography at the Paediatric Department of Lagos State University Teaching Hospital, Lagos Nigeria between January 2007 and December 2014. Results: Cardiomyopathy was documented in 31 (2.75\%) children with structural heart disease and constituted $25 \%$ of patients with acquired heart disease during the study period. The prevalence of cardiomyopathy amongst the children attending the study centre was 9 in 100,000 children over an 8 year period. The mean age of the children was 5.48 \pm 4.0 with male to female ratio of 1:1.30. Congestive cardiac failure was the commonest indication for echocardiography, in $48.38 \%$ of all the patients. Dilated cardiomyopathy was the most common disease accounting for $71 \%$ of the cardiomyopathies, followed by Hypertrophic cardiomyopathy (16.1\%) and Restrictive Cardiomyopathy (12.9\%) respectively. The subjects with dilated cardiomyopathy were younger than the subjects with hypertrophic cardiomyopathy and restrictive cardiomyopathy.

Conclusions: Cardiomyopathy is an important cause of congestive cardiac failure in children. Dilated cardiomyopathy is the predominant type of cardiomyopathy in our subjects followed by hypertrophic and restrictive cardiomyopathy. The subjects with dilated cardiomyopathy were younger than the subjects with hypertrophic cardiomyopathy and restrictive cardiomyopathy. Endomyocardial fibrosis is the predominant form of restrictive cardiomyopathy in our subjects.
\end{abstract}

Keywords: Cardiomyopathy; Children; Acquired; Congestive; Cardiac; Failure; Hypertrophic; Restrictive

\section{Introduction}

In Sub-Saharan Africa, preventable causes of death such as infectious disease, malnutrition and neonatal conditions still predominate [1]. However, non-communicable diseases otherwise called neglected diseases are also important causes of morbidity and mortality even in children [2]. Acquired heart diseases (AHD) such as myocarditis, cardiomyopathies and rheumatic heart diseases are important diseases responsible for morbidity and mortality in children [3,4]. Although the distribution of the AHD varies from region to region, in recent times myocarditis with dilated cardiomyopathy were the commonest acquired heart disease followed by pericarditis and rheumatic heart disease in children in Africa [5]. With the advent of echocardiography in many centers, more cases of cardiomyopathies are being recorded.

Cardiomyopathies are a heterogeneous group of diseases of the myocardium associated with mechanical and/or electrical dysfunction, which usually (but not invariably) exhibit inappropriate ventricular hypertrophy or dilatation, due to a variety of etiologies that frequently are genetic. Cardiomyopathies are either confined to the heart or are part of generalized systemic disorders, and often lead to cardiovascular death or progressive heart failure-related disability [6]. There is no general consensus on the classification of cardiomyopathies but traditionally, Cardiomyopathy has been classified as Dilated Cardiomyopathy (DCM), Restrictive Cardiomyopathy (RCM), Hypertrophic Cardiomyopathy (HCM), Arrhythmogenic Right Ventricular Cardiomyopathy (ARVC) and unclassified cardiomyopathies [7]. American Heart Association (AHA) has classified the cardiomyopathies as either primary or secondary [6]. The primary heart cardiomyopathies are those disorders that are predominantly confined to the heart muscle and they could be of genetic, non-genetic or acquired origin. Secondary cardiomyopathies are disorders that have myocardial damage as a result of systemic or multiorgan disease [6]. 
The management of patients with cardiomyopathies have many challenges especially in resource poor countries, some of which includes the difficulties in diagnosis which requires specialized cardiological investigations that are lacking in these regions, the lack of access to appropriate interventions such as heart transplantation and a high mortality associated with it [8].

There are have been only one reports from Nigeria on cardiomyopathy in children, the only one, reported only dilated cardiomyopathies in only six cases [9]. There has also been no previous reports on cardiomyopathy in children in Lagos hence the need for this study which aimed to describe the pattern and distribution of Cardiomyopathies in children less than 13 years in a tertiary hospital in Southwestern Nigeria and compare with patterns in the sub-region.

\section{Methods}

This is a review of prospectively collected data of all patients diagnosed with cardiomyopathy using clinical evaluation and echocardiography at the Paediatric Department of Lagos State University Teaching Hospital (LASUTH) Lagos Nigeria between January 2007 and December 2014.

The hospital is a tertiary institution in Southwestern Nigeria and receives referral from the region. The general Paediatric Department is an 83 bedded ward. The out-born unit is equipped with 14 cots and 12 incubators. A Paediatric Cardiologist is in charge of the cardiology unit. Patients referred to the cardiology unit of the Department are evaluated with chest radiograph, electrocardiography and echocardiography and other ancillary investigations as required.

One echocardiography machine was used on all the subjects throughout the study period, a GE Vivid Q echocardiography machine reference number 14502 WP SN 2084 with appropriate sized probe. It has facility for two dimensional, M-mode and color flow Doppler imaging. The Paediatric Cardiologist performed the echocardiography on all the subjects. Hypertrophic cardiomyopathy (HCM) was diagnosed on the basis of asymmetric septal hypertrophy, septal disorganization and systolic anterior motion of the anterior leaflet of MV, the ratio of septal to posterior wall thickness is greater than 1.3 [10,11]. Dilated cardiomyopathy was diagnosed from echocardiographic evidence, including at least two left ventricular measurements (decreased fractional shortening, decreased posterior wall thickness, or increased end-diastolic dimension) exceeding 2 standard deviations for age or for body surface area, the presence of dilated left atrium with poorly contractile left ventricle (left ventricular systolic or diastolic dysfunction) with LVEF less than 50\% [12]. The diagnosis of RCM was based on an echocardiographic pattern with "one or both atria enlarged relative to ventricles of normal or small size with evidence of impaired diastolic filling and in the absence of significant valvar heart disease and shortened mitral and tricuspid deceleration time $(<150 \mathrm{~ms})$ which is shortened further during inspiration $[11,13]$.

The data were imputed in a personal laptop and analysed using Statistical Package for Social Sciences (SPSS) version 20. The children's age, sex, indication for echocardiography and diagnosis were documented. Tables are used to depict those variables. Means of continuous variables were compared using the Student $t$ test and proportions using Chi-square test. Level of significance set at $\mathrm{p}<0.05$.

\section{Results}

A total of 315,150 children $\leq 13$ years were seen at the in-and out-patients units of the Department of Paediatrics, LASUTH during the study period out of which 1495 children had echocardiography done. 1126 (75.32\%) of the total children who had echocardiography done had structural heart disease. Cardiomyopathy was diagnosed in 31 (2.75\%) children with heart diseases (congenital and acquired) and $25 \%$ of those with acquired heart diease. Two out of every one hundred children who had echocardiography done had cardiomyopathy while a quater of those with acquired heart disease had cardiomyopathy. They comprised 14 males and 17 females with male to female ratio of 1:1.30. The mean age of the subjects was $5.48 \pm 4.0$ while for the females and males is $5.97 \pm 4.14$ and $4.85 \pm 3.89$ respectively. The difference in the mean age of the subject according to their gender is not statistically significant $(\mathrm{p}=0.46)$. The prevalence of cardiomyopathy amongst the children attending the Department of Paediatrics, LASUTH was 9 in 100,000 children over an 8 year period.

The indications for the echocardiography in the subjects is as shown in Table 1. The commonest indication for echocardiography was congestive cardiac failure comprising $48.38 \%$ of all the patients.

Dilated cardiomyopathy was the most common disease accounting for 71\% of the cardiomyopathies. Followed by HCM and RCM respectively as shown in Figure 1. Figure 2 illustrates the sex distribution of each type of cardiomyopathy in the subjects. The subjects with DCM were younger than both HCM and RCM, this difference was statistically significant for DCM and RCM ( $\mathrm{p}=$ $0.01)$, and while it was not statistically significant for DCM and HCM ( $\mathrm{p}=0.87)$.

Tables 2 depicts the distribution of cardiomyopathy in the study subjects. The relative frequencies, sex distribution and mean age of children with cardiomyopathies are displayed in Tables 3 below.

\section{Dilated cardiomyopathy}

There were 22 cases of DCM, comprising 71\% of all the cases of cardiomyopathies. It was diagnosed in 12 females and 10 males with a male to female ratio of 1:1.2. The children were between nine weeks and 11 years (mean of $4.76 \pm 3.53$ ) with a median age of 6.5. years. Twelve children were $\leq 5$ years, eleven were $\geq 5$ years and the age of one was not documented. 


\begin{tabular}{|c|c|c|}
\hline Indications & Number & percentage $(\%)$ \\
\hline Congestive cardiac failure & 14 & 45.2 \\
\hline Suspected Nephrotic syndrome & 2 & 6.5 \\
\hline Respiratory tract infection & 2 & 6.5 \\
\hline Suspected ACHD & 1 & 3.2 \\
\hline Retroviral disease with PTB & 1 & 3.2 \\
\hline ECG findings & 1 & 3.2 \\
\hline Chemotherapy induced Cardiomyopathy & 1 & 3.2 \\
\hline Breathlessness & 1 & 3.2 \\
\hline Murmur & 1 & 3.2 \\
\hline Tachycardia & 1 & 3.2 \\
\hline Palpitation & 1 & 3.2 \\
\hline Dysmorphology & 1 & 3.2 \\
\hline Ascites & 1 & 3.2 \\
\hline Others & 3 & 9.7 \\
\hline Total & 31 & 100ACHD- \\
\hline $\begin{array}{c}\text { Acyanotic congenital heart disease, } \\
\text { Tuberculosis }\end{array}$ & PTB- Pulmonary & \\
\hline
\end{tabular}

Table 1: Indications for echocardiography in the subjects

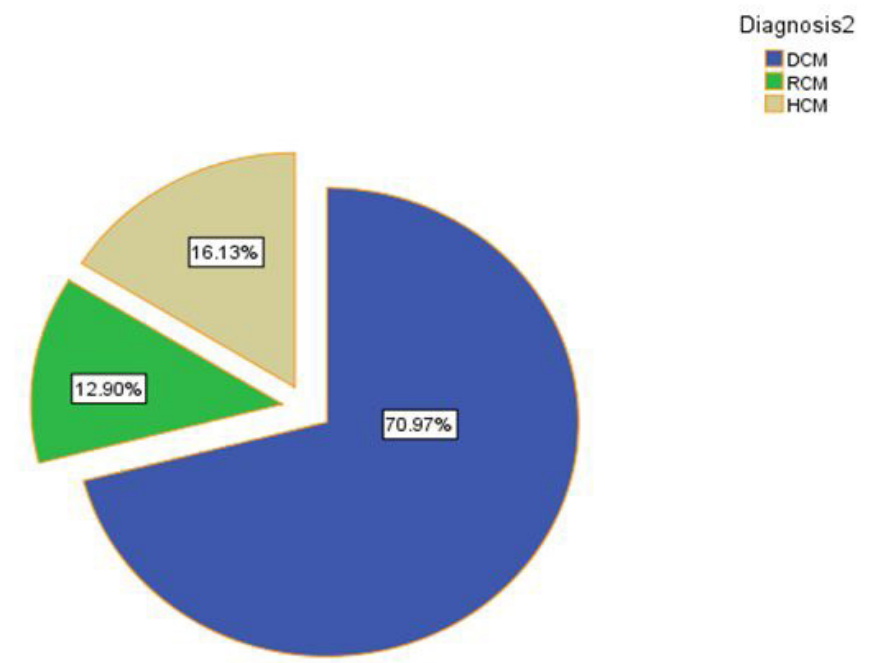

Figure 1: Distribution of Cardiomyopathy in the study subjects

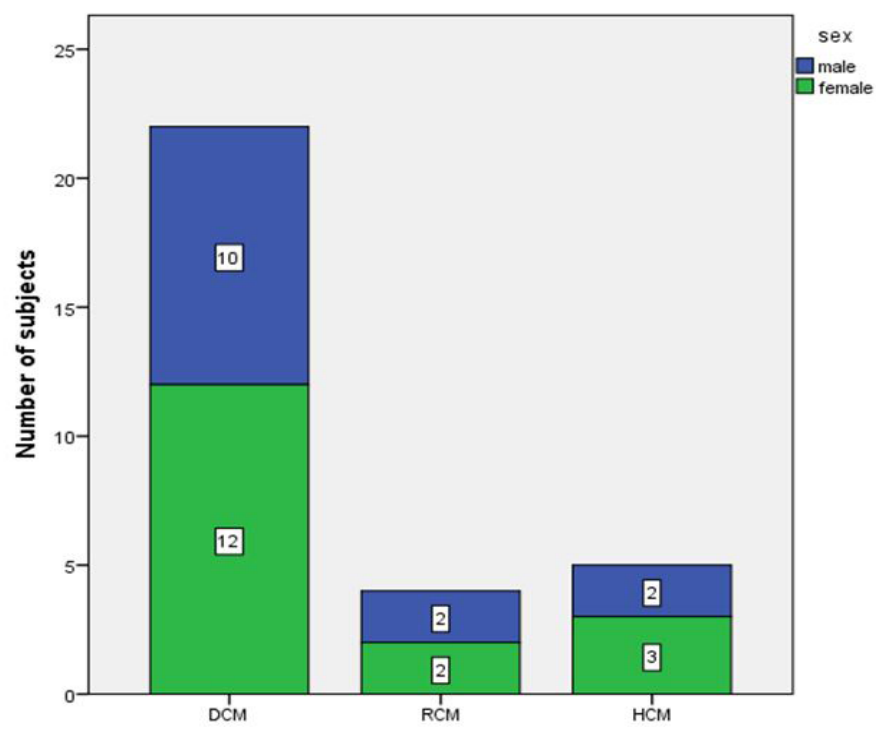

Figure 2: Sex distribution of the Cardiomyopathies 


\begin{tabular}{|c|c|c|}
\hline Cardiomyopathy & Mean age+SD & Age range \\
\hline DCM & $4.3 \pm 3.5$ & $0.17-11$ \\
\hline RCM & $10.0 \pm 2.94$ & $7-13$ \\
\hline HCM & $5.05 \pm 4.96$ & $0.25-10.58$ \\
\hline Total & $5.48 \pm 4.0$ & $0.17-13$ \\
\hline
\end{tabular}

Table 2: Ages in years at echocardiography diagnosis of the cardiomyopathies

\begin{tabular}{|c|c|c|c|c|c|c|}
\hline Age groups(Years) & Cardiomyopathy & Male n (\%) & Female n (\%) & Total & $\mathbf{X}^{2}$ & $\mathrm{P}$ \\
\hline \multirow{3}{*}{$0-4$} & DCM & $6(19.35)$ & $5(16.13)$ & $11(35.348)$ & \multirow{3}{*}{0.42} & \multirow{3}{*}{0.51} \\
\hline & $\mathrm{RCM}$ & 0 & 0 & 0 & & \\
\hline & $\mathrm{HCM}$ & $1(3.23)$ & $2(6.45)$ & $3(9.67)$ & & \\
\hline \multirow{3}{*}{$5-9$} & DCM & $2(6.45)$ & $6(19.35)$ & $8(25.81)$ & \multirow{3}{*}{5.0} & \multirow{3}{*}{0.08} \\
\hline & $\mathrm{RCM}$ & $2(6.45)$ & 0 & $2(6.45)$ & & \\
\hline & $\mathrm{HCM}$ & $0(0)$ & 0 & 0 & & \\
\hline \multirow{3}{*}{$\geq 10$} & DCM & $1(3.23)$ & $1(3.23)$ & $2(6.45)$ & \multirow{3}{*}{4.0} & \multirow{3}{*}{0.13} \\
\hline & $\mathrm{RCM}$ & 0 & $2(6.45)$ & $2(6.45)$ & & \\
\hline & $\mathrm{HCM}$ & $1(4.17)$ & $1(3.23)$ & $2(8.33)$ & & \\
\hline \multirow{3}{*}{ Total } & DCM & $9(29.03)^{*}$ & $12(38.71)$ & $22(71)$ & & \\
\hline & $\mathrm{RCM}$ & $2(6.45)$ & $2(6.45)$ & $4(12.90)$ & & \\
\hline & $\mathrm{HCM}$ & $2(6.45)$ & $3(9.67)$ & $5(16.1)$ & & \\
\hline
\end{tabular}

Table 3: Ages at echocardiography and sex distribution of children with Cardiomyopathy

\section{Restrictive cardiomyopathy}

There were 4 cases of RCM accounting for $12.9 \%$ of the cardiomyopathies in the study subjects, including three cases of endomyocardial fibrosis (EMF). There were equal numbers of males and females with a male to female ratio of 1:1 and the children were aged 7-13 years with a mean age of $10 \pm 2.94$.

\section{Hypertrophic cardiomyopathy}

HCM was diagnosed in five patients, representing $16.13 \%$ of the cardiomyopathies in the study subjects, with a male to female ratio of $1: 1.5$. The subjects were aged 3 months to 10 years 7 months with a mean age of $5.05 \pm 4.96$ years. Two of the subjects were infants, one was 3 years old and the remaining were $\geq 10$ years.

\section{Discussion}

This study aimed to describe a hospital based pattern of childhood cardiomyopathy in a tertiary health facility in South Western Nigeria. Cardiomyopathy was documented in $2.7 \%$ of all children under 13 years with a structural (acquired and congenital) heart lesions. This finding is similar to earlier reports [14,15]. Okoromah, et al. [14] documented seven cases of cardiomyopathy with a percentage of 2.4 over a two year period. Bode-Thomas, et al. [15] in their study on acquired heart disease in children in Jos, documented 37 cases of cardiomyopathy over a 10year period. This finding is similar to what was recorded in the present study (32 cases in eight years), the difference being the shorter duration of study in the present study and a fewer echocardiography done in the Jos study compared to the present study. The incidence of cardiomyopathy is difficult to describe in Africa because there are no community and population based studies and secondly because of a lack of uniform denominator in most descriptive studies. The incidence in developed countries is between 1.13 per 100,000 and 1.24 per 100,000 children [16,17]. The prevalence of cardiomyopathy in children in this study is 9 in 100,000 children. This value is much higher than that recorded in western countries $[16,17]$ the difference being that our study is hospital based and may not be a true reflection of the actual value in the general population when compared with the value in USA and Australia which is a population based study.

The most common cardiomyopathy was DCM accounting for $71 \%$ of all the cases. This is consistent with findings within and outside Africa [15-17]. The children with cardiomyopathy were aged 9 weeks to 11 years with a mean age of $4.96 \pm 3.53$. Not surprisingly, congestive cardiac failure was the most common mode of presentation in the subjects with DCM accounting for more than $50 \%$ of the presentation. This is also consistent with findings from other researchers $[5,9,16,18-20]$.

HCM was the second most common cardiomyopathy in this study. In other studies, HCM was also more common than RCM but less common than DCM $[5,9,16]$. HCM is a heterogeneous disease with diverse clinical presentation and course, presenting at different ages from infancy to the elderly. In this study, our subjects were aged 3months to over 10years and they presented with varied clinical presentation. The diagnosis of HCM was made clinically by cardiac imaging using a 2-dimensional echocardiograph. Genetic testing is used to make a definitive diagnosis in suspected familial cases. Genetic testing is not readily available and it is expensive making it inaccessible by the patients. There are was a case of a dysmorphic child with a possible genetic mutation, however there was no known familial tendency and genetic studies could not be done to exclude possible mutations in affected genes. 
RCM was the least common form of cardiomyopathy in this study. In the western countries, RCM is the least common of the cardiomyopathies $[9,14,15]$. In Africa, especially around the sub-tropical and tropical regions EMF, a form of RCM, is common. All our cases of RCM presented with features of EMF. Our subjects with RCM were aged 7-13 years with a mean age of $10 \pm 2.94$ years. Our finding is almost comparable with findings by Sadoh, et al. [5], where all cases presented with features of EMF with age range of 8-15 years. The mean age of this study subjects is slightly lower than that reported by Sadoh, et al. [5] because the subjects in this study were $\leq 13$ years while Sadoh, et al. included children up to 15 years. The mean age of our study subjects is higher than the mean age of $5.7 \pm 6.1$ years documented by Russo, et al. [21] in children with RCM in the western countries. Other researchers in Africa, Sliwa, et al. [8] also documented a higher age range, 11-15, compared with that recorded in western countries. The difference in the mean age between children in African compared to studies in the western countries may be due to the predominance of EMF in African children compared with the idiopathic RCM in the later and possibly related to early presentation and diagnosis in the former compared to the later. Three of the four cases were EMF, one case presented with right sided EMF and the remaining two with Biventricular involvement. The patient with right side EMF presented with massive ascites with no peripheral oedema while the other subjects had congestive cardiac failure and generalized oedema. EMF in Africa is more commonly right sided or biventricular and rarely left sided, [8] and this was what was seen in our study subjects.

In conclusion cardiomyopathy is an important cause of congestive cardiac failure in children. DCM is the predominant type of cardiomyopathy in our subjects followed by HCM and RCM. Children with DCM were younger than those with HCM and RCM albeit not statistically significant for HCM but significant in RCM. EMF is the predominant form of RCM in our subjects.

\section{Acknowledgement}

We gratefully acknowledge the children who participated in this study and their parents including other staff who were involved in their care.

\section{References}

1. WHO: Children; reducing mortality Sept 2014. Accessed February 2, 2015.

2. Miranda JJ, Kinra S, Casas JP, Davey Smith G, Ebrahim S (2008) Non-communicable diseases in low- and middle-income countries: context, determinants and health policy. Trop Med Int Health 13: 1225-34.

3. Silwa K, Mocumbi AO (2010) Forgotten cardiovascular diseases in Africa. Clin Res Cardiol 99: 65-74.

4. Mocumbi AO (2010) Neglected cardiovascular disease in Africa. Challenges and opportunities. J Am Coll Cardiol 55: 680-7.

5. Wilson SE, Chinyere UC, Queennette D (2014) Childhood acquired heart disease in Nigeria: an echocardiographic study from three centres. Afr Health Sci 14: 609-16.

6. Maron BJ, Towbin JA, Thiene G, Antzelevitch C, Corrado D, et al. (2006) Contemporary Definitions and Classification of Cardiomyopathies: an American Heart Association Scientific Statement From the Council On Clinical Cardiology, heart Failure and Transportation Committee; Quality of Care and Outcomes Research and Functional Genomics and Translational Biology Interdisciplinary Working Groups: and Council on Epidemiology and Prevention. Circulation 113: 1807-16.

7. Singh BK, Pillai KK, Kohli K, Haque SE (2012) Classification and definitions of cardiomyopathies, Cardiomyopathies-From basic research to clinical management, Prof. Veselka J (editor), InTech.

8. Sliwa K, Damasceno A, Mayosi BM (2005) Epidemiology and etiology of cardiomyopathy in Africa. Circulation 112: $3577-83$.

9. Bode-Thomas F, Ebonyi AO, Animasahun BA (2005) Childhood dilated cardiomyopathy in Jos, Nigeria. Sahel Med J 8: 100-5.

10. Elliott PM, Anastasakis A, Borger MA, Borggrefe M, Cecchi F (2014) 2014 ESC Guidelines on diagnosis and management of hypertrophic cardiomyopathy. European Heart 2733-79.

11. Snider R (1997) Cardiomyopathies. In: Snider R, Serwer G, Ritter S, editors. Echocardiography in Pediatric Heart Disease. United States of America: Walsworth. $2^{\text {nd }}$ ed. $512-29$

12. Harmon WG, Sleeper LA, Cuniberti L, Messere J, Colan SD (2009) Treating Children with Idiopathic Dilated Cardiomyopathy (From the Pediatric Cardiomyopathy Registry). Am J Cardiol 104: 281-6.

13. Webber SA, Lipshultz S, Sleeper LA, Lu M, Wilkinson JD (2012) Outcomes of Restrictive Cardiomyopathy in Childhood and the Influence of Phenotype: A Report From the Pediatric Cardiomyopathy Registry. Circulation 126: 1237-44.

14. Okoromah CA, Ekure EN, Ojo OO, Animasahun BA, Bastos MI (2008) Structural heart disease in children in Lagos: profile, problems and prospects. Niger Postgrad Med J 15: 82-8.

15. Bode-Thomas F, Ige OO, Yilgwan C (2013) Childhood acquired heart diseases in Jos, North Central Nigeria. Niger Med J 54: 51-8.

16. Lipshultz SE, sleeper LA, Towbin JA, Lowe AM, Orav EJ, et al. (2003) The incidence of pediatric cardiomyopathy in two regions of the United States. N Eng J Med 348: 1647-55.

17. Nugent AW, Dauberry PE, Chondros P, Carlon JB, Cheungí M, et al. (2003) The epidemiology of childhood cardiomyopathy in Australia. N Eng J Med 348: 1639-46.

18. Towbin JA, Lowe AM, Colan SD, Sleeper LA, Orav EJ, et al. (2006) Incidence, causes and outcomes of dilated cardiomyopathy in children. JAMA 296: 1867-76.

19. Antia AU, Cockshot WP, Thorpe GJ (1969) Idiopathic cardiomegaly in Nigerian children. Br Heart J 31: 178-83.

20. Malcic I, Jelusic M, Kniewald H, Barisic N, Jelasic D, et al. (2002). Epidemiology of cardiomyopathies in children and adolescents: a retrospective study over the last 10 years. Cardiol Young 12: 253-9.

21. Russo LM, Webber SA (2005) Idiopathic restrictive cardiomyopathy in children. Heart 91: 1199-202. 


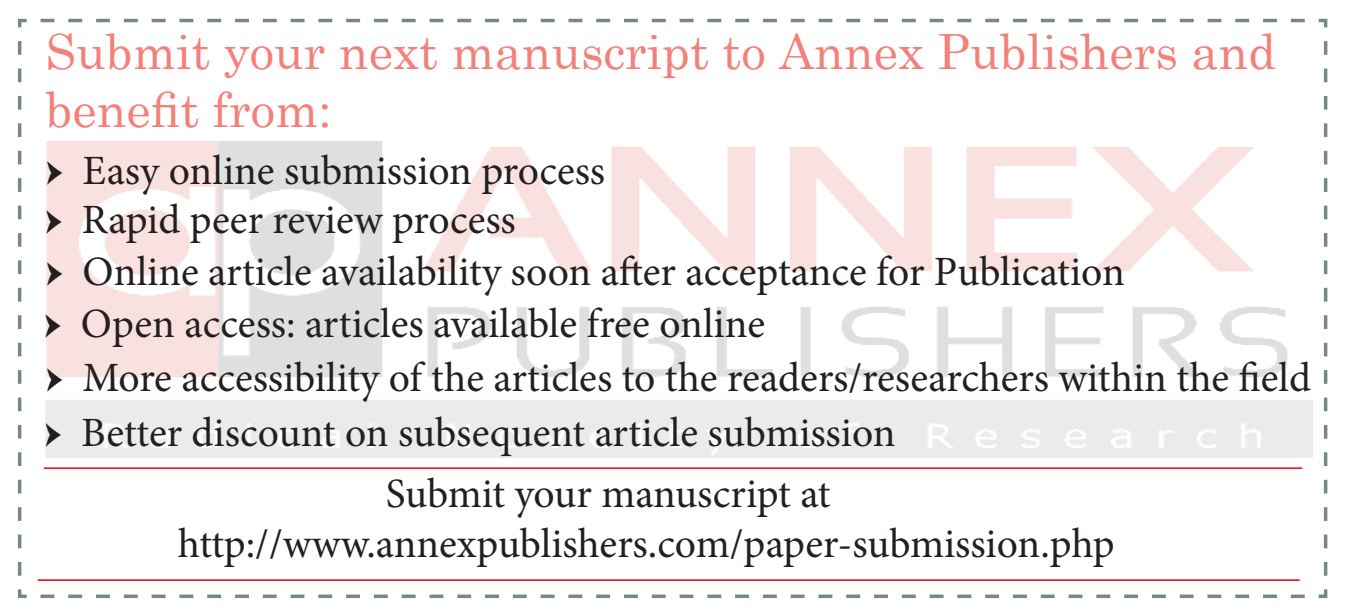

